CLINICAL STUDY

\title{
Discrepant results in the diagnosis of GH deficiency with the insulin-tolerance test and the GHRH plus arginine test in patients with traumatic brain injury
}

\author{
H J Schneider, B L Herrmann ${ }^{1}$, M Schneider, C Sievers, L Schaaf and G K Stalla \\ Internal Medicine, Endocrinology and Clinical Chemistry, Max Planck Institute of Psychiatry, Kraepelinstr, 10, D-80804 Munich, Germany ${ }^{1}$ Division of \\ Endocrinology, Department of Internal Medicine, University Hospital of Essen, Essen, Germany \\ (Correspondence should be addressed to H J Schneider; Email: schneider@mpipsykl.mpg.de)
}

\begin{abstract}
Objective: Patients with traumatic brain injury (TBI) are at moderate risk of GH deficiency (GHD), requiring a diagnostic test with high specificity. The GHRH + arginine (GHRH + ARG) test has been recommended as a reliable alternative to the insulin-tolerance test (ITT) as a standard test with a cutoff level of $9 \mathrm{ng} / \mathrm{ml}$. However, it has recently been questioned for its low specificity in obese subjects, and now BMI-dependent cut-off levels are available. In this study, we compared the ITT and GHRH+ ARG test in patients with TBI.

Design: A cross-sectional study

Methods: We performed an ITT and a GHRH + ARG test in 21 patients with TBI ( 6 women, 15 men; mean age $40.2 \pm 12.1$ years; BMI $30.7 \pm 6.2$ ). The number of patients classified discordantly as $\mathrm{GH}$ deficient by the ITT and the GHRH + ARG test with both classical and BMI-dependent cut-off levels was assessed.

Results: Using the GHRH + ARG test with the classical cut-off $(\leq 9 \mathrm{ng} / \mathrm{ml})$, we identified 12 patients as GH deficient who had a normal GH response to ITT ( $>3 \mathrm{ng} / \mathrm{ml})$, and one patient as GH sufficient who had a blunted GH response to ITT (discordance rate 61.9\%). All patients discordantly classified as GH deficient by the GHRH + ARG test had a BMI of $\geq 28$. With the BMI-dependent cut-offs $(4.2,8.0$, and $11.5 \mathrm{ng} / \mathrm{ml}$ in obese, overweight, and lean subjects respectively), only 3 of the 21 patients were discordantly classified (discordance rate 14.3\%).

Conclusions: Our results discourage the use of a cut-off level of $9 \mathrm{ng} / \mathrm{ml}$ for the GHRH+ARG test in obese subjects. The diagnostic reliability of this test is improved with the BMI-dependent cut-offs.
\end{abstract}

European Journal of Endocrinology 155 553-557

\section{Introduction}

Recent studies have stressed the role of traumatic brain injury (TBI) as a potential cause of growth hormone (GH) deficiency (GHD) reviewed in (1). However, as opposed to patients with pituitary-hypothalamic diseases and multiple pituitary deficits, who are at high risk of GHD, the risk of GHD ranges from 10 to $20 \%$ in TBI patients (1). In these patients, a test with a high specificity is necessary for the diagnosis of GHD to avoid a very high number of false-positive results (2). The insulin-tolerance test (ITT) has been shown to distinguish well between healthy subjects and patients with GHD (3) and has been recommended as the test of choice for the diagnosis of GHD by the growth hormone research society (4). Here, severe GHD was suggested to be defined at a cut-off level of $3 \mathrm{ng} / \mathrm{ml}$ or lower. However, this test is contraindicated in patients with several conditions, such as heart diseases and seizure disorders and causes more discomfort than other tests.
Ghigo et al. (5) suggested to use the GH-releasing hormone plus arginine (GHRH + ARG) test as a safe and sensitive alternative. They established a cut-off level of $9 \mathrm{ng} / \mathrm{ml}$ for the diagnosis of severe GHD in a lean population (5). However, recently, the GHRH + ARG test has been criticized to be strongly BMI dependent and to cause false-positive results in healthy obese and overweight subjects $(6,7)$. In TBI patients, GH levels decrease after GHRH+ARG stimulation, even after exclusion of obese subjects (8). Recently, Corneli et al. (9) have published BMI-dependent cut-off levels for the GHRH + ARG test. Another study, conducted mainly in an obese population, has suggested cut-off levels of 5.1 and $4.1 \mathrm{ng} / \mathrm{ml}$ for the ITT and GHRH + ARG test respectively (10). Yet, the cut-off of $9 \mathrm{ng} / \mathrm{ml}$ is still used in most endocrinological centers worldwide, irrespective of BMI, and this cut-off has been recommended for the diagnosis of GHD in recent reviews and consensus guidelines for both patients with known pituitary diseases and TBI $(11,12)$. 
In this study, we wanted to compare the ITT and the GHRH + ARG test in patients with TBI with regard to both the classical and the new BMI-dependent cutoff levels.

\section{Subjects and methods}

\section{Subjects}

We studied 21 consecutive patients with TBI ( 6 women, 15 men; age (mean \pm s.D.) $40.2 \pm 12.1$ years; BMI $30.7 \pm 6.2$; time after trauma $9.5 \pm 8.7$ years). Fourteen patients were evaluated in the neuroendocrine outpatient clinic of the Max Planck Institute of Psychiatry. In these patients, ITT and GHRH + ARG test were done during routine endocrine evaluations and ITT was done after GHRH + ARG test either as a confirmation test in case of low or borderline low-GH response to GHRH+ ARG, or, to exclude undetected hypothalamic deficiencies. All patients gave informed consent. Another seven patients were studied during a study of hypopituitarism after TBI in the University Hospital of Essen (13). These patients gave written informed consent and the study was approved by the local ethics committee.

\section{Endocrine evaluations}

We did a GHRH + ARG test and an ITT in all patients on 2 different days. All tests were performed in the morning after an overnight fast. For the ITT, $0.15 \mathrm{U} / \mathrm{kg}$ regular insulin (Actrapid Novo-Nordisk, Denmark), was given intravenously as a bolus at $0 \mathrm{~min}$. Blood samples were taken every $15 \mathrm{~min}$ from -15 to $+90 \mathrm{~min}$. During ITT, glucose measurement was performed, and minimum plasma glucose level of $2.2 \mathrm{mmol} / \mathrm{l}$ or less was detected together with hypoglycemic symptoms. If necessary, a second bolus was added to achieve adequate hypoglycemia. For the GHRH + ARG test, different protocols were used in the two centers. In the Max Planck Institute, $50 \mu \mathrm{g}$ GHRH (Ferring) were given intravenously as a bolus and $30 \mathrm{~g} \mathrm{~L}$-arginine in $250 \mathrm{ml}$ physiological salt solution were administered as a 30-min infusion, and GH was measured at $0,30,45$, 60,90 , and $120 \mathrm{~min}$. This protocol is routinely used in this center as it was shown that synthetic GH-releasing factor causes a dose-dependent $\mathrm{GH}$ response up to a dose of $50 \mu \mathrm{g}$ in healthy subjects, but no further increase of GH response to doses of 50-200 $\mu \mathrm{g}$ (14). In the University Hospital of Essen, $1 \mu \mathrm{g}$ GHRH/kg was administered by an i.v. bolus, followed by a 30-min infusion of $30 \mathrm{~g}$ arginine with the measurement of $\mathrm{GH}$ at $-15,0,15,30,45,60,75,90,105$, and $120 \mathrm{~min}$. Further hormone deficiencies were recorded by standard clinical procedures of the respective centers $(8$, 13). To compare the two tests, we considered both the classical cut-offs of $\leq 3$ and $\leq 9 \mathrm{ng} / \mathrm{ml}$ for the ITT $(3,4)$ and GHRH + ARG test (5) respectively, and the
BMI-dependent cut-off levels with $\leq 11.5, \leq 8.0$, and $\leq 4.2 \mathrm{ng} / \mathrm{ml}$ in lean $(\mathrm{BMI}<25)$, overweight $(\mathrm{BMI} \geq 25$ to $<30$ ), and obese (BMI $\geq 30$ ) subjects respectively (9). Additionally, we also considered the cut-offs of $\leq 5.1$ and $\leq 4.1 \mathrm{ng} / \mathrm{ml}$ for the ITT and GHHR + ARG respectively as suggested by Biller et al. (2002). For insulin-like growth factor (IGF)-I levels, age-dependent SDS were calculated (15).

GH and IGF-I were measured by chemiluminiscence with the Nichols Advantage system (Nichols Institute Diagnostics, San Clemente, CA, USA). The maximal intra- and inter-assay coefficients of variation at different hormone concentrations were as follows: $\mathrm{GH}$, $8 \%$ and $12 \%$; IGF-I, $5 \%$ and $7 \%$ respectively.

\section{Statistical analyses}

Data are presented as mean \pm S.D. We analyzed the numbers and percentages classified concordantly and discordantly by the two tests using the different cut-off values. To test for significance between groups, we used the Mann-Whitney U-test. Correlations were calculated with the Spearman's correlations coefficient. A $P$ value $<0.05$ was considered significant.

\section{Results}

Mean peak GH levels after ITT and GHRH + ARG test were $11.6 \pm 10.5$ and $15.0 \pm 19.9 \mathrm{ng} / \mathrm{ml}$ respectively. BMI correlated negatively with peak GH after GHRH + ARG test $(r=0.66, P=0.001)$, but not after ITT $(r=-0.21, \quad P=0.35)$. These findings remained unchanged after adjustment for age.

Table 1 summarizes the results and clinical characteristics of the patients. The number of patients with peaks below the respective cut-off values is shown in Table 2 . Only three patients $(14.3 \%)$ had a GH response below or equal to the cut-off of $3 \mathrm{ng} / \mathrm{ml}$ to ITT, whereas 14 patients $(66.7 \%)$ had GH responses $\leq 9 \mathrm{ng} / \mathrm{ml}$ to GHRH+ARG stimulation. Two patients had IGF-I levels $<2$ s.D. below the age-related normal values. Both of these had a peak $\mathrm{GH} \leq 3 \mathrm{ng} / \mathrm{ml}$ after ITT. Table 3 shows the number of patients classified concordantly or discordantly by the two tests using different cut-off values. The discordance rate was the highest when using the classical cut-offs of 3 and $9 \mathrm{ng} / \mathrm{ml}$ for the ITT and GHRH + ARG test respectively $(61.9 \%)$ and the lowest when using $3 \mathrm{ng} / \mathrm{ml}$ for the ITT and the BMI-dependent cut-offs for the GHRH + ARG (14.3\%). The one patient identified as GH deficient with the ITT and not the GHRH + ARG test, using classical cutoffs, had panhypopituitarism, hyperprolactinemia, and an IGF-I level of $21 \mathrm{ng} / \mathrm{ml}(<-4$ s.D. $)$. If we analyzed the patients of the clinical centers separately using the classical cut-offs, 8 of 14 patients from the Max Planck Institute and five out of seven patients from the University Hospital of Essen were classified discordantly (discordance rates $57.1 \%$ and $71.4 \%$ respectively). In detail, at the 
Table 1 Patient characteristics.

\begin{tabular}{|c|c|c|c|c|c|c|c|}
\hline Patient & Sex & BMI & Age & Peak GH ITT & $\begin{array}{c}\text { Peak GH } \\
\text { GHRH+ARG }\end{array}$ & IGF-1 SDS & $\begin{array}{l}\text { Other deficits/hormone } \\
\text { disturbances }\end{array}$ \\
\hline 1 & w & 17.0 & 24 & 0.9 & 10.8 & -4.22 & $\begin{array}{l}\mathrm{LH} / \mathrm{FSH}, \mathrm{ACTH}, \mathrm{TSH} \text {, } \\
\text { hyperprolactinemia }\end{array}$ \\
\hline 2 & w & 18.4 & 44 & 8.2 & 81.6 & -1.54 & $\mathrm{n}$ \\
\hline 3 & $\mathrm{~m}$ & 23.1 & 21 & 25.9 & 40.6 & -0.14 & ACTH \\
\hline 4 & w & 26.0 & 41 & 38.5 & 33.9 & 0.52 & ACTH \\
\hline 5 & $\mathrm{~m}$ & 27.1 & 56 & 14.1 & 14.8 & 0.20 & $\mathrm{n}$ \\
\hline 6 & $\mathrm{~m}$ & 27.7 & 46 & 33.5 & 47.7 & -1.06 & LH/FSH \\
\hline 7 & $\mathrm{~m}$ & 28.0 & 31 & 25.3 & 8.8 & -1.29 & $\mathrm{LH}$, hyperprolactinemia \\
\hline 8 & $\mathrm{~m}$ & 28.7 & 57 & 8.1 & 7.1 & -0.82 & $\mathrm{n}$ \\
\hline 9 & $\mathrm{~m}$ & 30.0 & 58 & 1.5 & 3.8 & -0.93 & ACTH \\
\hline 10 & $\mathrm{~m}$ & 30.5 & 39 & 5.2 & 5.5 & -0.91 & $\mathrm{n}$ \\
\hline 11 & $\mathrm{~m}$ & 30.6 & 24 & 13.2 & 5.0 & -0.95 & LH/FSH, ACTH \\
\hline 12 & $\mathrm{~m}$ & 31.0 & 16 & 3.1 & 7.0 & 1.02 & $\mathrm{LH}$ \\
\hline 13 & $\mathrm{~m}$ & 33.0 & 49 & 9.1 & 5.0 & -0.15 & $\mathrm{n}$ \\
\hline 14 & $\mathrm{~m}$ & 34.0 & 47 & 2.2 & 2.8 & -2.07 & $\mathrm{n}$ \\
\hline 15 & w & 34.6 & 41 & 4.2 & 5.5 & -0.62 & $n$ \\
\hline 16 & $\mathrm{~m}$ & 35.0 & 37 & 4.8 & 7.4 & -0.86 & $\mathrm{n}$ \\
\hline 17 & $\mathrm{~m}$ & 36.3 & 50 & 13.9 & 5.6 & -0.25 & $\mathrm{LH} / \mathrm{FSH}$ \\
\hline 18 & $\mathrm{~m}$ & 37.7 & 43 & 5.4 & 1.9 & NA & $\mathrm{n}$ \\
\hline 19 & $\mathrm{~m}$ & 38.0 & 34 & 9.5 & 6.7 & 0.13 & $\mathrm{LH} / \mathrm{FSH}$ \\
\hline 20 & w & 38.2 & 53 & 9.2 & 10.2 & -0.43 & $\mathrm{n}$ \\
\hline 21 & w & 39.5 & 34 & 7.9 & 3.5 & -1.18 & $\mathrm{n}$ \\
\hline
\end{tabular}

BMI, body mass index; GH, growth hormone; ITT, insulin tolerance test; GHRH + ARG, GH-releasing hormone + arginine test; IGF-I, insulin-like growth factorI; w, women; m, men; LH, luteinizing hormone; FSH, follicle-stimulating hormone; ACTH, adrenocorticotropic hormone; TSH, thyroid-stimulating hormone; $\mathrm{NA}$, not available; $n$, no deficit/hormone disturbance.

Table 2 Numbers and percentage of patients with peak GH levels below the respective cut-offs.

\begin{tabular}{lccccc}
\hline & \multicolumn{5}{c}{ GHRH + ARG } \\
\cline { 2 - 6 } ITT & $\leq 3 \mathrm{ng} / \mathrm{ml}$ & $\leq 5.1 \mathrm{ng} / \mathrm{ml}$ & $\leq 9 \mathrm{ng} / \mathrm{ml}$ & $\leq$ BMI-dependent cut-off & $\leq 4.1 \mathrm{ng} / \mathrm{ml}$ \\
\hline $\mathrm{n}$ & 21 & 3 & 6 & 14 & 6 \\
$\%$ & 100 & 14.3 & 28.6 & 66.7 & 4 \\
\hline
\end{tabular}

ITT, insulin tolerance test; GHRH + ARG, GH-releasing hormone+arginine test; BMI, body mass index.

University Hospital of Essen, five patients were classified discordantly as GH deficient by the GHRH + ARG only, and two were classified concordantly GH deficient by both tests. At the Max Plack Institute, seven patients were identified discordantly by the GHRH + ARG test only and one patient by the ITT only.

Figure 1 depicts the association of peak responses to GHRH + ARG test and ITT in lean, overweight, and obese subjects and the different cut-offs of the respective tests. Of the 12 patients discordantly classified as $\mathrm{GH}$ deficient by the GHRH + ARG test only using the classical cut-offs, ten were obese. The other two patients (patients 7 and 8) were overweight with BMIs of 28.0 and 28.7. Only one of all obese subjects had a peak GH above $9 \mathrm{ng} / \mathrm{ml}$ after GHRH + ARG test.

There were no significant differences among age, time after trauma, IGF-I, or IGF-I SDS between obese and non-obese subjects.

Table 3 Numbers of patients classified as GH deficient by both tests (concordant classification) or only one test (discordant classification) using different cut-offs.

\begin{tabular}{lcccc}
\hline Cut-off used for definition of GHD & $\begin{array}{c}\text { GHD both } \\
\text { tests }\end{array}$ & $\begin{array}{c}\text { GHD only } \\
\text { ITT }\end{array}$ & $\begin{array}{c}\text { GHD only } \\
\text { GHRH+ARG }\end{array}$ & $\begin{array}{c}\% \text { Discordant } \\
\text { classification }\end{array}$ \\
\hline$\leq 3 \mathrm{ng} / \mathrm{ml}($ ITT) vs $\leq 9 \mathrm{ng} / \mathrm{ml}(\mathrm{GHRH}+\mathrm{ARG})$ & 2 & 1 & 12 & 61.9 \\
$\leq 3 \mathrm{ng} / \mathrm{ml}(\mathrm{ITT})$ versus $\leq$ BMl-dependent cut-off (GHRH+ARG) & 3 & 0 & 3 & 14.3 \\
$\leq 5.1 \mathrm{ng} / \mathrm{ml}($ ITT) versus $\leq \mathrm{BMl}-$ dependent cut-off (GHRH+ARG) & 3 & 3 & 3 & 28.6 \\
$\leq 5.1 \mathrm{ng} / \mathrm{ml}$ (ITT) vs $\leq 4.1 \mathrm{ng} / \mathrm{ml}$ (GHRH+ARG) & 2 & 4 & 2 & 28.6
\end{tabular}

GHD, growth hormone deficiency; ITT, insulin tolerance test; GHRH +ARG, GH-releasing hormone + arginine test. 


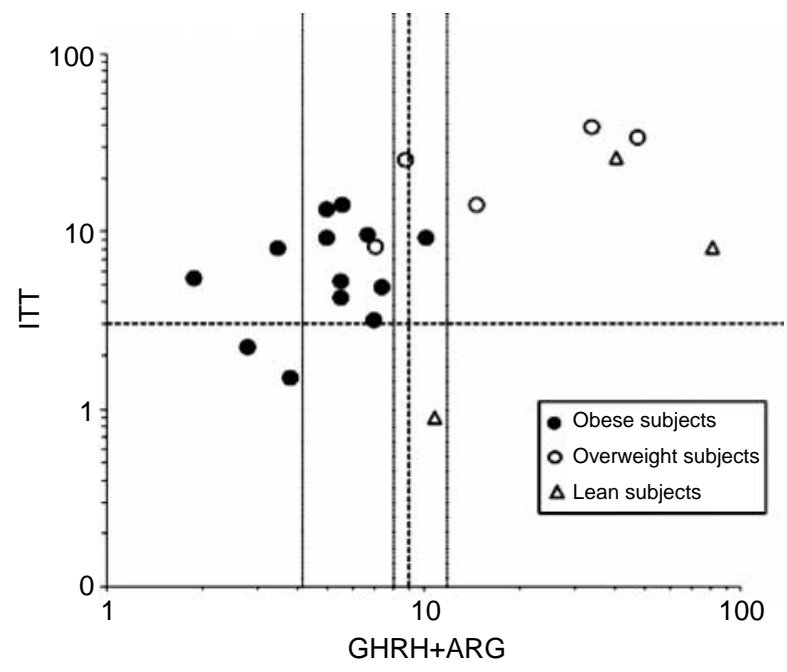

Figure 1 Correlation of peak growth hormone $(\mathrm{GH})$ responses to insulin tolerance test (ITT) and GH-releasing hormone+ arginine (GHRH+ARG) test. The perforated lines indicate the classical cut-off levels for ITT and GHRH+ARG test. The dotted lines indicate the cut-off levels for obese, overweight, and lean subjects (Corneli et al. 2005). Please note that scaling is logarithmic and axes do not start at 0 .

\section{Discussion}

In this study, we have compared two stimulation tests for the diagnosis of GHD in identical patients with TBI, a condition that has only recently been highlighted to be at substantial risk of GHD. Our study shows that: (a) the majority of patients are classified as GH deficient with the GHRH + ARG test but not the ITT; (b) all of these patients have a BMI of 28 or higher; (c) there is a clear BMI dependence of the GHRH + ARG test but not the ITT; and (d) the concordance of the tests is increased if the BMI-dependent cut-offs are used.

TBI can cause pituitary dysfunction in $30-50 \%$ of patients $(1,8)$ and there is now a consensus that endocrine assessment should be implemented in the routine follow-up of TBI patients $(16,17)$. The GHRH + ARG test has been suggested as a safe and reliable alternative to the ITT (5) and the cut-off of $9 \mathrm{ng} / \mathrm{ml}$ is commonly used worldwide to diagnose severe GHD. Our data show a strong BMI dependence of this test in TBI patients and confirm similar findings from healthy subjects $(6,7)$. This leads to a large proportion of obese subjects classified as GH deficient using the classical cutoff for the GHRH + ARG test, even though a normal GH secretion is shown with the ITT. Thus, we must assume that these patients are misclassified as GH deficient. Therefore, if the decision to initiate $\mathrm{GH}$ replacement was done on the basis of the GHRH + ARG test with a cut-off of $9 \mathrm{ng} / \mathrm{ml}$, the large majority of obese TBI patients would receive unjustified GH therapy.

The discordance rate is lower using the cut-offs suggested by Biller et al. (10) and lowest if the BMIdependent cut-offs are used for the GHRH + ARG test, although some patients were still discordantly classified.
Even though we found no clear BMI dependence of the ITT, we cannot rule out that this was due to small patient number or too few normal weight subjects. Qu et al. (7) have shown BMI dependence of the ITT in healthy subjects, albeit less strong than for the GHRH + ARG test. Also BMI-dependent cut-offs may increase the accuracy of this test but they are not available. Moreover, theoretically, it is also possible that the ITT causes falsenegative results. The high BMI in our population reflects the typical population of patients with TBI seen in our endocrine outpatient clinics. Most patients gain significant weight after TBI. Thus, it is of particular importance to take the possible effects of obesity into account when testing endocrine function in these patients.

The sensitivity and specificity of diagnostic testing increase if two or more different tests, instead of merely one test, are used. Therefore, a higher concordance rate of different tests indicates a higher accuracy of the single tests. Taking the above-mentioned limitations into account, the lower discordance rate indicates reasonable accuracy of the two tests.

It can be criticized that different protocols used for stimulation testing in the two centers might have influenced the results. However, this seems unlikely as the discordance rates in both centers were very similar. One patient with panhypopituitarism and hyperprolactinemia, indicative of hypothalamic dysfunction or stalk dysruption was not identified as GH deficient by a cut-off of $9 \mathrm{ng} / \mathrm{ml}$ with the GHRH + ARG test. This shows that the GHRH + ARG test is less sensitive than the ITT for suprapituitary causes of dysfunction in these patients.

In summary, our data discourage the use of the classical cut-offs for the GHRH + ARG test in obese and maybe overweight patients for the diagnosis of GHD. The BMI-dependent cut-offs lead to better results, even though some patients with normal GH response to ITT may be classified as GH deficient with this test. Additionally, the GHRH + ARG test may be less sensitive to hypothalamic dysfunction. In clinical practice, the decision to initiate GH substitution should be ideally based on more than one adequate stimulation test with appropriate cut-offs in synopsis with clinical symptoms, additional deficiencies and IGF-I levels.

\section{References}

1 Schneider M, Schneider HJ \& Stalla GK. Anterior pituitary hormone abnormalities following traumatic brain injury. Journal of Neurotrauma 200522 937-946.

2 Kreitschmann-Andermahr I, Schneider HJ \& Saller B. Growth hormone deficiency after brain injury-induced hypopituitarism: how should it be diagnosed? Journal of Clinical Endocrinology and Metabolism 2005 e-Letter.

3 Hoffman DM, O'Sullivan AJ, Baxter RC \& Ho KK. Diagnosis of growthhormone deficiency in adults. Lancet 1994343 1064-1068.

4 Anonymous. Consensus guidelines for the diagnosis and treatment of adults with growth hormone deficiency: summary 
statement of the growth hormone research society workshop on adult growth hormone deficiency. Journal of Clinical Endocrinology and Metabolism $1998 \mathbf{8 3} 379-381$.

5 Ghigo E, Aimaretti G, Gianotti L, Bellone J, Arvat E \& Camanni F. New approach to the diagnosis of growth hormone deficiency in adults. European Journal of Endocrinology 1996134 352-356.

6 Bonert VS, Elashoff JD, Barnett P \& Melmed S. Body mass index determines evoked growth hormone $(\mathrm{GH})$ responsiveness in normal healthy male subjects: diagnostic caveat for adult $\mathrm{GH}$ deficiency. Journal of Clinical Endocrinology and Metabolism 200489 3397-3401.

7 Qu XD, Gaw Gonzalo IT, Al Sayed MY, Cohan P, Christenson PD, Swerdloff RS, Kelly DF \& Wang C. Influence of body mass index and gender on growth hormone $(\mathrm{GH})$ responses to GH-releasing hormone plus arginine and insulin tolerance tests. Journal of Clinical Endocrinology and Metabolism 200590 1563-1569.

8 Schneider HJ, Schneider M, Saller B, Petersenn S, Uhr M, Husemann B, von Rosen F \& Stalla GK. Prevalence of anterior pituitary insufficiency 3 and 12 months after traumatic brain injury. European Journal of Endocrinology $2006154259-265$.

9 Corneli G, Di Somma C, Rovere R, Gasco V, Croce CG, Grottoli S, Maccario M, Colao A, Lombardi G, Ghigo E, Camanni F \& Aimaretti G. The cut-off limits of the GH response to GH-releasing hormone-arginine test related to body mass index. European Journal of Endocrinology 2005153 257-264.

10 Biller BM, Samuels MH, Zagar A, Cook DM, Arafah BM, Bonert V, Stavrou S, Kleinberg DL, Chipman JJ \& Hartman ML. Sensitivity and specificity of six tests for the diagnosis of adult GH deficiency. Journal of Clinical Endocrinology and Metabolism 200287 2067-2079.

11 van Aken MO \& Lamberts SW. Diagnosis and treatment of hypopituitarism: an update. Pituitary 2005 8 183-191.
12 Ghigo E, Masel B, Aimaretti G, Leon-Carrion J, Casanueva FF, Dominguez-Morales MR, Elovic E, Perrone K, Stalla G, Thompson C \& Urban R. Consensus guidelines on screening for hypopituitarism following traumatic brain injury. Brain Injury 200519 711-724.

13 Herrmann BL, Rehder J, Kahlke S, Wiedemayer H, Doerfler A, Ischebeck W, Laumer $R$, Forsting $M$, Stolke D \& Mann K. Hypopituitarism following severe traumatic brain injury. Experimental and Clinical Endocrinology and Diabetes 2006114 316-321.

14 Losa M, Stalla GK, Müller OA \& von Werder K. Human pancreatic growth hormone-releasing factor (hpGRF): dose-response of GRFand GH-levels. Klinische Wochenschrift 198361 1249-1253.

15 Brabant G, von zur Mühlen A, Wüster C, Ranke MB, Kratzsch J, Kiess W, Ketelslegers JM, Wilhelmsen L, Hulthen L, Saller B, Mattsson A, Wilde J, Schemer R \& Kann P. German KIMS board. Serum insulin-like growth factor I reference values for an automated chemiluminescence immunoassay system: results from a multicenter study. Hormone Research 200360 53-60.

16 Ghigo E, Masel B, Aimaretti G, Leon-Carrion J, Casanueva FF, Dominguez-Morales MR, Elovic E, Perrone K, Stalla G, Thompson C \& Urban R. Consensus guidelines on screening for hypopituitarism following traumatic brain injury. Brain Injury $200519711-724$.

17 Schneider HJ, Stalla GK \& Buchfelder M. Expert meeting: hypopituitarism after traumatic brain injury and subarachnoid haemorrhage. Acta Neurochirurgica $2006 \mathbf{1 4 8} 449-456$.

Received 21 June 2006

Accepted 17 July 2006 